\title{
Hepatitis B virus vaccination booster does not provide additional protection in adolescents: a cross-sectional school-based study
}

\author{
Yung-Chieh Chang ${ }^{1 \dagger}$, Jen-Hung Wang ${ }^{2 \dagger}$, Yu-Sheng Chen ${ }^{1 \dagger}$, Jun-Song Lin ${ }^{1 \dagger}$, Ching-Feng Cheng ${ }^{1,2,3+}$ \\ and Chia-Hsiang Chu ${ }^{1 *}$
}

\begin{abstract}
Background: Current consensus does not support the use of a universal booster of hepatitis B virus (HBV) vaccine because there is an anamnestic response in almost all children 15 years after universal infant HBV vaccination. We aimed to provide a booster strategy among adolescents as a result of their changes in lifestyle and sexual activity.

Methods: This study comprised a series of cross-sectional serological surveys of HBV markers in four age groups between 2004 and 2012. The seropositivity rates of hepatitis B surface antigen (HBsAg) and its reciprocal antibody (anti-HBs) for each age group were collected. There were two parts to this study; age-specific HBV seroepidemiology and subgroup analysis, including effects of different vaccine types, booster response for immunogenicity at 15 years of age, and longitudinal follow-up to identify possible additional protection by HBV booster.

Results: Within the study period, data on serum anti-HBs and HBsAg in a total of 6950 students from four age groups were collected. The overall anti-HBs and HBsAg seropositivity rates were $44.3 \%$ and $1.2 \%$, respectively. The anti-HBs seropositivity rate in the plasma-derived subgroup was significantly higher in both 15- and 18-year age groups. Overall response rate in the double-seronegative recipients at 15 years of age was $92.5 \%$ at 6 weeks following one recombinant HBV booster dose. Among the 24 recipients showing anti-HBs seroconversion at 6 weeks after booster, seven subjects (29.2\%) had lost their anti-HBs seropositivity again within 3 years. Increased seropositivity rates and titers of anti-HBs did not provide additional protective effects among subjects comprehensively vaccinated against HBV in infancy.

Conclusions: HBV booster strategy at 15 years of age was the main contributor to the unique age-related phenomenon of anti-HBs seropositivity rate and titer. No increase in HBsAg seropositivity rates within different age groups was observed. Vaccination with plasma-derived HBV vaccines in infancy provided higher anti-HBs seropositivity at 15-18 years of age. Overall booster response rate was $92.5 \%$ and indicated that intact immunogenicity persisted at least 15 years after primary HBV vaccination in infancy. Booster vaccination of HBV did not confer additional protection against HBsAg carriage in our study.
\end{abstract}

Keywords: HBV booster, Adolescents, Anamnestic response, Infant HBV vaccination

\footnotetext{
*Correspondence: chuchia@ms6.hinet.net

'Equal contributors

'Department of Pediatrics, Hualien Tzu Chi Hospital, Buddhist Tzu Chi

Medical Foundation, Hualien, Taiwan

Full list of author information is available at the end of the article
} 


\section{Background}

The world's first nationwide hepatitis B virus (HBV) infant vaccination program was launched in Taiwan in July 1984, starting with newborns of highly infectious mothers and expanding to all newborns in July 1986 [1]. Prior to July 1992, infants were given four doses of plasma-derived vaccine at birth, 1, 2, and 12 months of age. After July 1992, three doses of recombinant vaccine were administered at the age of less than 1 week, 1 month, 6 months [2]. The protective cut-off level was set at $\geq 10 \mathrm{mIU} / \mathrm{mL}$ for antibody to hepatitis B surface antigen (anti-HBs) based on vaccine efficacy studies [3]. Over the past 20 years, the hepatitis B surface antigen ( $\mathrm{HBsAg}$ ) seropositivity rate has decreased from $9.8 \%$ in 1984 to $0.6 \%$ in 2004 among people younger than 20 years of age in Taipei, Taiwan [4-7]. Despite the success of the universal infant hepatitis $B(H B)$ vaccination program, chronic $\mathrm{HBV}$ infection and hepatocellular carcinoma were not eliminated in children in Taiwan. Among the children who initially responded to the primary three-dose vaccination series, $15-50 \%$ demonstrate a low or undetectable anti-HBs level 5-15 years after primary vaccination [8]. Although perinatal hepatitis $B$ virus transmission is still the main cause for vaccine failure [2], horizontal and breakthrough infection may also occur after waning or eventual loss of vaccine protectiveness in older children, especially with changes in lifestyle and sexual activity [9]. Currently, a booster of $\mathrm{HB}$ vaccination is not recommended for the general healthy population after primary immunization because of the absence of increased HBsAg seropositivity at different ages $(<20$ years of age), which implies that there is no increased risk of persistent $\mathrm{HBV}$ infection with aging [7]. Over the years, the role of the anamnestic response, indicating immune memory to $\mathrm{HBsAg}$, was confirmed after anti-HBs levels had decreased to below the seroprotective level. However, a large-scale study provided evidence that an anamnestic anti-HBs response was absent in $10.1 \%$ of 15 - to 18 -yearold individuals in Taiwan, a country that had high endemicity of HBV [10].

In this report, we describe two parts of the present study; age-specific HBV seroepidemiology and subgroup analysis including effects of different vaccine types, immunogenicity response to booster at 15 years of age, and longitudinal follow-up to assess possible additional protection by HBV booster.

\section{Methods}

\section{Vaccination program in Taiwan}

The nationwide $\mathrm{HBV}$ infant vaccination program in Taiwan began with vaccination of newborns of highly infectious mothers in July 1984 and then expanded to all newborns in July 1986 [1]. Before July 1992, four doses (5 $\mu \mathrm{g} /$ dose) of plasma-derived vaccine were given at birth, 1, 2, and 12 months of age. After July 1992, three doses of recombinant vaccine were given at the age of less than 1 week, 1 month, and 6 months [2]. After July 1991 (birth cohort 1986), all newly enrolled elementary school first graders were required to provide their vaccination cards for mandatory check-up, and those children with incomplete vaccination records were given catch-up HBV vaccination before enrolment.

\section{Study population}

This was a retrospective cross-sectional study composed of serological surveys of HBV markers between 2004 and 2012 from newly enrolled students of the Tzu-Chi University-affiliated education system, including elementary school (birth cohort 1998-2006), junior high (birth cohort 1992-1994), senior high (birth cohort 1989-1997) schools, and university (birth cohort 19861994) in Eastern Taiwan. A flow chart indicating our study design is depicted in Figure 1. An approval certificate for this study was also issued from the Research Ethics Committee of Hualien Tzu Chi Hospital, Buddhist Tzu Chi Medical Foundation (REC No.: IRB101-125). A total of 16,110 records including anti-HBs or HBsAg were included in our database. We excluded subjects without records of paired HBV markers (anti-HBs/ HBsAg) and those who were born before January 1st 1987, leaving a total of 6950 subjects in the epidemiological study. We hypothesized that all of our subjects received HBV vaccination within about 6 months following the introduction of the new vaccination policy at that time. Based on the statistical records of Taiwan National Immunization Information System (NIIS), the immunization coverage rates of complete HBV vaccination were $88.8-97.7 \%$ (birth cohort 1984-2013), and the complete rate of $\mathrm{HBV}$ vaccination among elementary school enrollments was above 99\% after the 1997 birth cohort $[11,12]$. Written consent forms that were provided by the Hualien County Government Education Bureau were obtained from the students' parents or guardians upon school enrollment. The consent form informed parents/guardians that these examinations were non-intrusive with minimum risk and students or parent/guardians were free to withdraw from any examination item at any time.

\section{Epidemiological study}

There were four groups of enrolled students: first graders in elementary school (age 6 years), junior and senior high school students (age 12 years and age 15 years) and freshmen at university (age 18 years). Blood samples were collected from each student as part of their health examination during the first semester of their enrollment. The rates of anti-HBs and HBsAg seropositivity among each group were collected and the data spanning all 9 years were analyzed. In addition to 


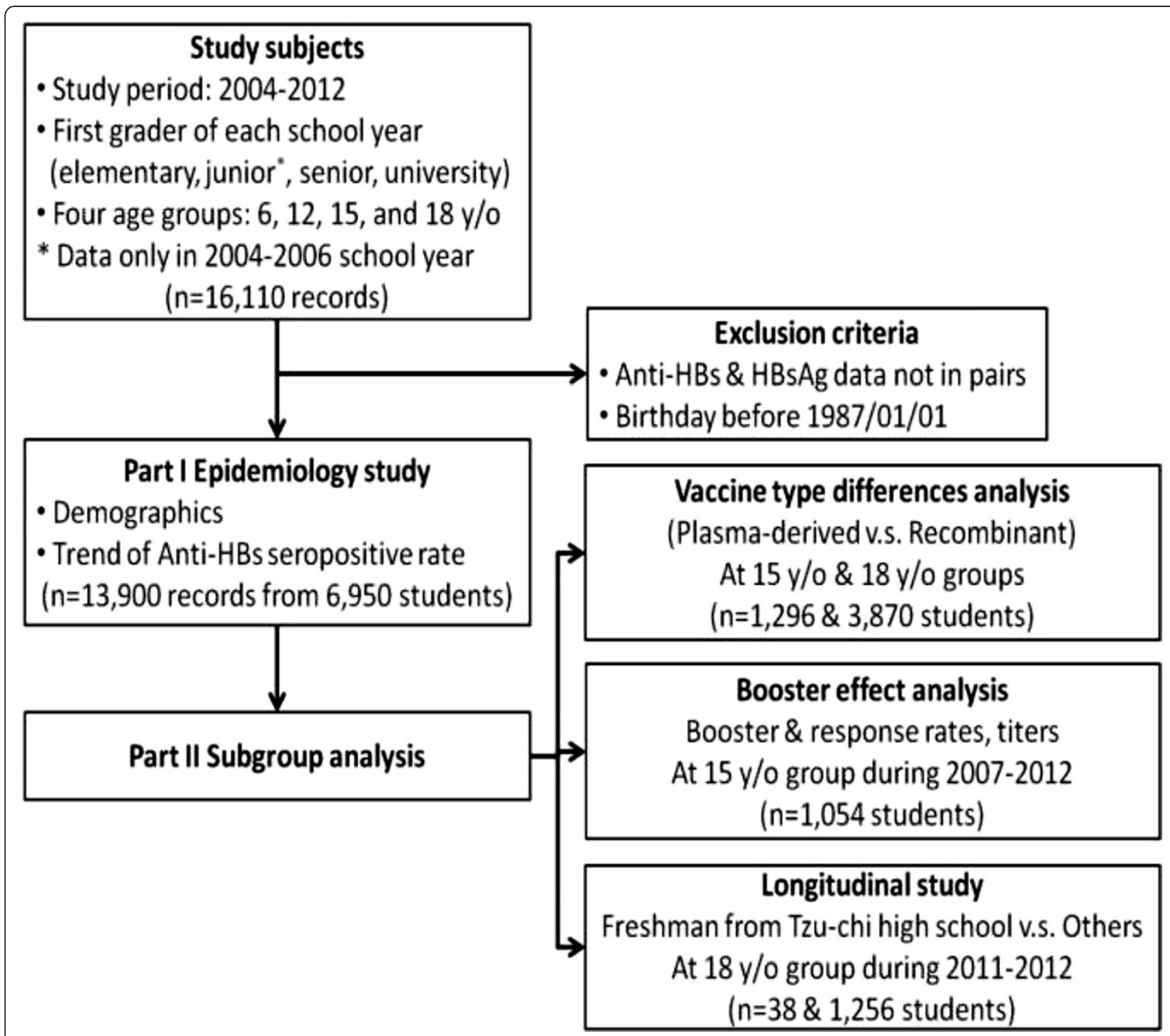

Figure 1 Flow chart of study design.

qualitative anti-HBs data, anti-HBs serum titers were available after 2007 for all school stages in our education system. The rates of anti-HBs and HBsAg seropositivity, along with other demographic characteristics such as gender, age, and median titers of anti-HBs were also compared among different age groups. In the 12 -year age group, only data from the school years 2004-2006 were available in our study. Therefore, we also investigated whether there were any differences in demographic characteristics within different time spans such as 2004-2006 versus 2004-2012. There were no data for anti-HBc available within our database. Therefore, prevalence rates of natural infection could not be estimated in our study.
Subgroup analysis

Comparison of HBV markers by different vaccine types

In our study subjects, we noted some were vaccinated with a plasma-derived HBV vaccine and others received recombinant $\mathrm{HBV}$ vaccine in infancy, based on their birth cohort. Subjects born before July 1992 received four doses of plasma-derived vaccine (Hevac B; Pasteur-Merieux, or its equivalent derivative); others, who were born after July 1992, received recombinant $\mathrm{HBV}$ vaccines $(5 \mu \mathrm{g} /$ dose of Recombivax [Merck] or $20 \mu \mathrm{g} /$ dose of Engerix [GSK]) [1]. Therefore, we selected subjects by their birth cohort, grouping them as either plasma-derived or recombinant vaccine recipients. In the 15-year age group, subjects from birth cohorts 1989-1991 and 1993-1997 were grouped as 
plasma-derived and recombinant groups, respectively. In the 18-year age group, subjects from birth cohorts 19871991 and 1993-1994 were defined as plasma-derived and recombinant groups, accordingly. We compared the seroprevalence of anti-HBs and HBsAg following administration of the different vaccine types in these two age groups.

\section{Immunogenicity response in booster recipients at 15 years of age}

In our database, subjects in birth cohort 1992-1997 demonstrating double seronegativity for anti-HBs and HBsAg in the 15-year age group were recommended to receive one booster dose of recombinant $\mathrm{HBV}$ vaccine $(20 \mu \mathrm{g} / \mathrm{dose})$. One booster dose was to be administered in their school clinic on the same day under the supervision of the family physician. Post-booster blood sampling for anti-HBs titers were also performed on the same day in the school clinic an average of 6 weeks (range 35-50 days) after their booster date. The response rate to one booster dose of HBV vaccine was defined as the proportion of booster recipients whose post-booster titer was $\geq 10 \mathrm{mIU} / \mathrm{mL}$. The overall and annual anti-HBs seropositivity rates after one booster dose of HBV vaccine were also calculated. Anti-HBs seropositivity rates after one booster dose were defined by the following equation:

\section{Post-booster anti-HBs seropositive rate $=$ pre-booster anti-HBs seropositive rate$$
+ \text { (pre-booster anti-HBs seronegative rate) }
$$$$
\times(\text { booster rate }) \times(\text { response rate })
$$

This equation was modified from the formula by $\mathrm{Wu}$ et al. [13]. In their study, the overall anti-HBs seropositive rate, designated as $\mathrm{PR}_{\mathrm{T}}$, after a booster dose of $\mathrm{HB}$ vaccine was approximated by the following formula:

$$
\mathrm{PR}_{\mathrm{T}}=\mathrm{PR}_{1}+\left(1-\mathrm{PR}_{1}\right) \times \mathrm{PR}_{2},
$$

where $\mathrm{PR}_{1}$ is the anti-HBs seropositive rate the before booster, and $\mathrm{PR}_{2}$ is the response rate in the booster recipients.

All of the seronegative subjects had received a booster dose in the Wu et al. study [13]. Therefore, no booster rate was needed in their formula. In our study, the booster rate was defined as the percentage of seronegative subjects receiving one booster dose. The response rate was defined as the proportion of those booster recipients whose post-booster anti-HBs titer was $\geq 10 \mathrm{mIU} / \mathrm{mL}$.

\section{Longitudinal study}

In our database of the university-affiliated education system, we found by birth cohort tracing, that some of our subjects studied in our senior high school and then consequently in our university. We decided to follow the subjects of birth cohort 1993-1994 in the 18-year age group who had received the recombinant $\mathrm{HBV}$ vaccine in infancy. In this way, the effects of different vaccine type were eliminated. If the subjects previously studied in our senior high school (15-year age group, birth cohort 1993-1994), they were grouped as "the same school". Their serum data of both anti-HBs and HBsAg were analyzed longitudinally to trace the pattern of seroconversion and post-booster change in this 3-year interval. The other subjects of the 18year age group from the 1993-1994 birth cohort were used as the comparison group, which was labeled as "the others". No records of booster history in "the others" group was available, therefore, an uncertain proportion of subjects in this group may have received a booster dose after their HBV vaccination in infancy. We hypothesize that median value of anti-HBs before HBV booster among subjects in birth cohort 1993-1994 in the 15-year age group could serve as the baseline median value in the 18-year age group. The actual median titers of anti-HBs in the 18-year age group would be further decayed if a booster dose was not given or natural exposure did not occur. In addition to the seropositivity rate of anti-HBs and $\mathrm{HBsAg}$, median values of anti-HBs titers at 18 years of age were compared among two groups, "the same school" versus "the others", to identify if booster effects were present in our comparison group. We aimed to identify any additional protective effect by increasing the levels of anti-HBs titers by comparing the HBsAg seropositivity rate between these two groups.

\section{Serologic testing}

All quantification of seromarkers of HBV infection was performed by enzyme immunoassay (data prior to 2011/08/31, VITROS ECiQ Immunodiagnostics system, Ortho Clinical Diagnostics; data after 2011/09/01, Abbott Laboratories, North Chicago, IL, USA). Reference concentrations from the WHO were used as criteria values and the protective level of anti-HBs was defined as $\geq 10 \mathrm{mIU} / \mathrm{mL}$ [3]. Subjects who were positive for HBsAg were assumed to be hepatitis B carriers [14].

\section{Statistical analysis}

A Chi-square test was performed to identify the differences in seropositivity rates, including anti-HBs and HBsAg, median titers of anti-HBs, between different ages, genders, and other subgroups. Statistically significant differences were defined as $\mathrm{p}<0.05$. All of the statistical analyses were performed using SPSS software (version 17.0; SPSS Inc., Chicago, IL, USA).

\section{Results}

\section{Part I: epidemiological study}

The study subjects of this epidemiological survey consisted of 6950 individuals (2901 were male and 4049 were female), all of whom were born after 1987, within the national infant HBV vaccination era of Taiwan. There were 524, 450, 
1464, and 4512 individuals in the 6-, 12-, 15-, and 18-year age groups, respectively. The overall seropositivity rate of anti-HBs and HBsAg was $44.3 \%$ and $1.2 \%$, respectively, in our study subjects. Figure 2 shows the trend of anti-HBs seropositivity rate and median titers by different age group and time frame. Table 1 shows a statistically significant difference between age and the seropositivity rate of anti-HBs, with a declining rate from 6 years to 12 years of age, which then rises through 18 years of age in our study $(\mathrm{p}<0.001)$. However, the age-specific pattern of HBsAg seropositivity did not show any statistical significance $(\mathrm{p}=0.154)$ with aging. The median titers of anti-HBs also showed statistical significance with a pattern similar to that observed for antiHBs seropositivity. To assess the possible effects of a shortage of data within the 12-year age group from 2007 to 2012, we analyzed the seropositive rates of anti-HBs and HBsAg in all four age groups within the same interval (2004-2006). A similar age-related pattern of anti-HBs seropositivity was noted with a statistically significant difference among all individuals $(\mathrm{p}<0.001)$. We further pooled the two younger and two older age groups (6-12 vs 15-18 years) for comparison, and the results showed a similar pattern to the four age group comparison. As compared with seroprevalence data of HBV markers by genders of all subjects, female individuals had a significantly higher anti-HBs seropositivity rate $(\mathrm{p}<0.009)$ than male individuals, but this was not mirrored by HBsAg ( $=0.439)$.

\section{Part II: subgroup assessment}

\section{Comparison of HBV markers by different vaccine types}

In this part of our study, we selected the subjects by their birth cohort, grouping as plasma-derived and recombinant groups in two age groups. We compared the seroprevalence of anti-HBs and HBsAg within the different vaccine types in these two age groups. Table 2 shows that the seropositivity rate of anti-HBs in the plasma-derived subgroup was significantly higher in both the 15- and 18-year age groups $(\mathrm{p}<0.004$ and 0.003 , respectively). The seropositivity rate of $\mathrm{HBsAg}$ in the plasma-derived subgroup was also significantly higher in the 18-year age group $(\mathrm{p}=0.049)$, but not in the 15-year age group $(\mathrm{p}=0.129)$.

\section{Immunogenicity response in booster recipients in the 15-year age group}

Among 1054 subjects of birth cohort 1992-1997 in the 15year age group, 657 subjects (62.3\%) demonstrating seronegativity for both anti-HBs and HBsAg were further recommended to receive one booster dose of recombinant HBV vaccine $(20 \mu \mathrm{g} / \mathrm{dose})$. Table 3 shows that $570(86.8 \%)$ of these 657 double seronegative subjects received one booster dose and the overall response rate among booster recipients with post-booster titer $\geq 10 \mathrm{mIU} / \mathrm{mL}$ was $92.5 \%$ (529/570). The overall anti-HBs seropositivity rate before one booster dose was $37.7 \%$ (397/1054), while the overall anti-HBs seropositivity rate after one booster dose was $87.7 \%$. The annual post-booster rate had a range of 83.493.6\% in the 1992-1997 birth cohort. The median titers of anti-HBs before and after booster dose were $1.1 \mathrm{mIU} / \mathrm{mL}$ and $545.5 \mathrm{mIU} / \mathrm{mL}$, respectively.

\section{Longitudinal study}

Among 1294 subjects in the 18-year age group of the 1993-1994 birth cohort, only 38 (2.9\%) subjects had studied in our senior high school (15-year age group, birth cohort 1993-1994), followed by attendance at our
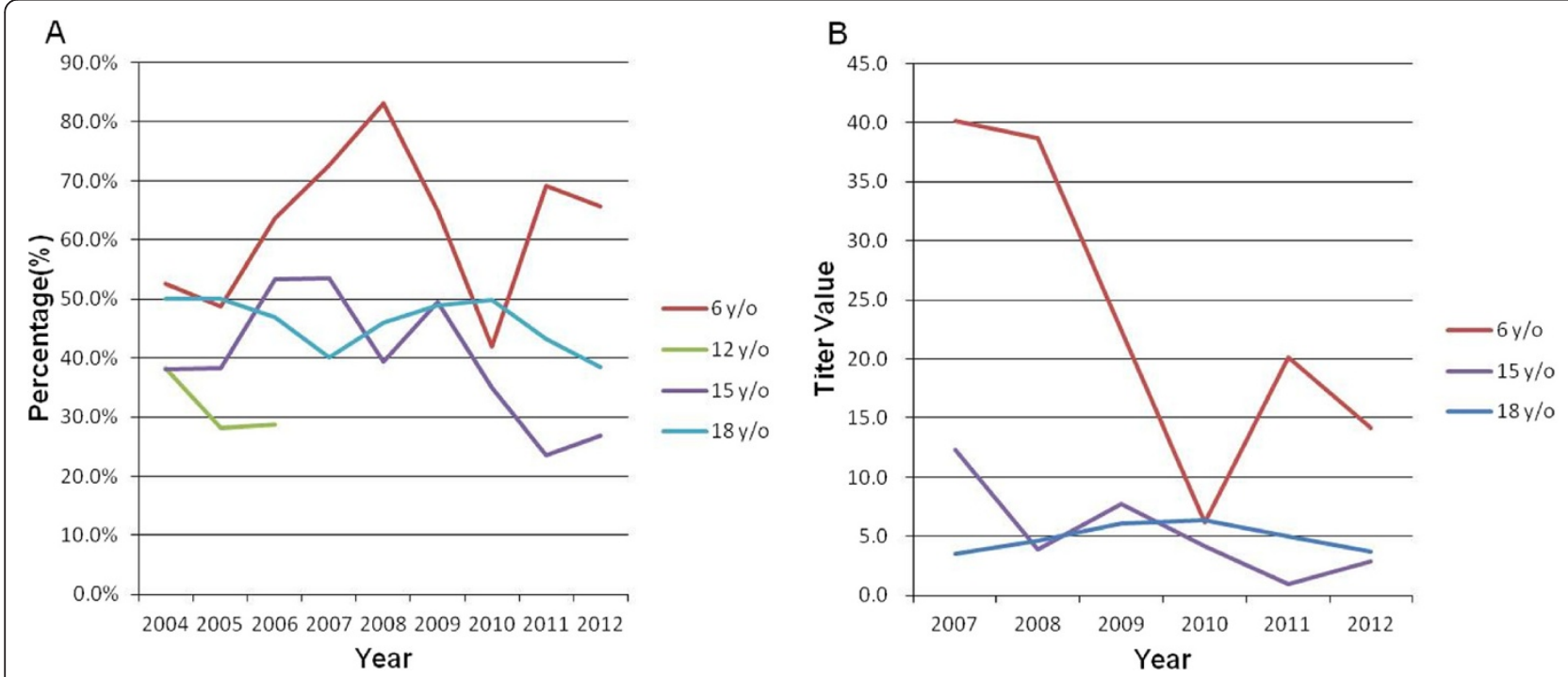

Figure 2 The trend of anti-HBs seropositivity rate and median titers by different age group and time frame. (A) The trend of anti-HBs seropositive rate by age (2004-2012). (B) The trend of median titer value of anti-HBs by age (2007-2012). 
Table 1 Demographics data and seroprevalence of hepatitis B virus (HBV) markers and titers

\begin{tabular}{|c|c|c|c|c|c|c|}
\hline $\begin{array}{l}\text { Age group } \\
\text { Item }\end{array}$ & $\begin{array}{c}6 y / 0 \\
(n=524)\end{array}$ & $\begin{array}{c}12 y / o \\
(n=450)\end{array}$ & $\begin{array}{c}15 y / o \\
(n=1464)\end{array}$ & $\begin{array}{c}18 y / o \\
(n=4512)\end{array}$ & $\begin{array}{l}6-12 y / o \\
(n=974)\end{array}$ & $\begin{array}{l}15-18 y / o \\
(n=5976)\end{array}$ \\
\hline \multicolumn{7}{|l|}{ Gender } \\
\hline Male, n (\%) & 299(57.1) & 205(45.6) & $740(50.5)$ & 1657(36.7) & $504(51.7)$ & 2397(40.1) \\
\hline Female, n (\%) & $225(42.9)$ & $245(54.4)$ & $724(49.5)$ & 2855(63.3) & 470(48.3) & $3579(59.9)$ \\
\hline Age (Mean $\pm S D, y r)$ & $6.5 \pm 0.3$ & $12.5 \pm 0.3$ & $15.5 \pm 0.5$ & $18.7 \pm 0.7$ & $9.3 \pm 3.0$ & $17.9 \pm 1.5$ \\
\hline Anti-HBs (+), n & 332 & 142 & 574 & 2033 & 474 & 2607 \\
\hline$(\%, 95 \% \mathrm{Cl})$ & $(63.4,59.3-67.5)$ & $(31.6,27.3-35.9)$ & $(39.2,36.7-41.7)$ & $(45.1,43.6-46.6)$ & $(48.7,45.6-51.8)$ & $(43.6,42.3-44.9)$ \\
\hline Anti-HBs (-), n & 192 & 308 & 890 & 2479 & 500 & 3369 \\
\hline$(\%, 95 \% \mathrm{Cl})$ & $(36.6,32.5-40.7)$ & $(68.4,64.1-72.7)$ & $(60.8,58.3-63.3)$ & $(54.9,53.4-56.4)$ & $(51.3,48.2-54.4)$ & $(56.4,55.1-57.7)$ \\
\hline P-value & \multicolumn{4}{|c|}{$<0.001^{*}$} & \multicolumn{2}{|c|}{$0.003^{*}$} \\
\hline Anti-HBs median titer & 21.2 & NA & 3.9 & 4.8 & 21.2 & 4.6 \\
\hline P-value & \multicolumn{4}{|c|}{$<0.001^{*}$} & \multicolumn{2}{|c|}{$<0.001^{*}$} \\
\hline $\operatorname{HBsAg}(+), n$ & 1 & 7 & 16 & 56 & 8 & 72 \\
\hline$(\%, 95 \% \mathrm{Cl})$ & $(0.2,0.0-0.6)$ & $(1.6,0.4-2.8)$ & $(1.1,0.6-1.6)$ & $(1.2,0.9-1.5)$ & $(0.8,0.2-1.4)$ & $(1.2,0.9-1.5)$ \\
\hline $\operatorname{HBsAg}(-), n$ & 523 & 443 & 1448 & 4456 & 966 & 5904 \\
\hline$(\%, 95 \% \mathrm{Cl})$ & $(99.8,99.4-100.0)$ & $(98.4,97.2-99.6)$ & $(98.9,98.4-99.4)$ & $(98.8,98.5-99.1)$ & $(99.2,98.6-99.8)$ & $(98.8,98.5-99.1)$ \\
\hline P-value & \multicolumn{4}{|c|}{0.154} & \multicolumn{2}{|c|}{0.336} \\
\hline
\end{tabular}

Note: Anti-HBs, antibody to HBV surface antigen; HBsAg, HBV surface antigen; y/o, year old; NA, not available; $95 \% \mathrm{Cl}, 95 \%$ confidence interval; Data are presented as or $\mathrm{n}$ and percentage.

${ }^{*} \mathrm{p}$-value $<0.05$ was considered statistically significant after test.

Table 2 Comparison of seroprevalence of hepatitis B virus (HBV) markers by different age and vaccine type received in infancy

\begin{tabular}{|c|c|c|c|c|c|}
\hline Age group & $\begin{array}{c}\text { Vaccine type } \\
\text { Items }\end{array}$ & Plasma-derived & Recombinant & Total & P-value \\
\hline \multirow{9}{*}{$15 \mathrm{y} / \mathrm{o}$} & $\mathrm{N}$ [birth cohort] & 410 [1989-91] & 886 [1993-97] & 1296 & \multirow{5}{*}{$0.004^{*}$} \\
\hline & anti-HBs (+), n & 177 & 307 & 484 & \\
\hline & $(\%, 95 \% \mathrm{Cl})$ & $(43.2,38.4-48.0)$ & $(34.7,31.6-37.8)$ & $(37.3,34.3-39.9)$ & \\
\hline & anti-HBs $(-), n$ & 233 & 579 & 812 & \\
\hline & $(\%, 95 \%$ Cl) & $(56.8,52.0-61.6)$ & $(65.3,62.2-68.4)$ & $(62.7,60.1-65.3)$ & \\
\hline & HBsAg $(+), n$ & 7 & 6 & 13 & \multirow{4}{*}{0.129} \\
\hline & $(\%, 95 \%$ Cl) & $(1.7,0.4-3.0)$ & $(0.7,0.2-1.2)$ & $(1.0,0.5-1.5)$ & \\
\hline & $\operatorname{HBsAg}(-), n$ & 403 & 880 & 1283 & \\
\hline & $(\%, 95 \%$ Cl) & $(98.3,97.0-99.6)$ & $(99.3,98.8-99.8)$ & $(99.0,98.5-99.5)$ & \\
\hline \multirow{9}{*}{$18 \mathrm{y} / \mathrm{o}$} & N [birth cohort] & 2575 [1987-91] & 1295 [1993-94] & 3870 & \multirow{5}{*}{$0.003^{*}$} \\
\hline & anti-HBs $(+), n$ & 1184 & 529 & 1713 & \\
\hline & $(\%, 95 \% \mathrm{Cl})$ & $(46.0,44.1-47.9)$ & $(40.8,38.1-43.5)$ & $(44.3,42.7-45.9)$ & \\
\hline & anti-HBs (-), n & 1391 & 766 & 2157 & \\
\hline & $(\%, 95 \% \mathrm{Cl})$ & $(54.0,52.1-55.9)$ & $(59.2,56.5-61.9)$ & $(55.7,54.1-57.3)$ & \\
\hline & $\operatorname{HBsAg}(+), n$ & 40 & 10 & 50 & \multirow{4}{*}{$0.049^{*}$} \\
\hline & $(\%, 95 \%$ Cl) & $(1.6,1.1-2.1)$ & $(0.8,0.3-1.3)$ & $(1.3,0.9-1.7)$ & \\
\hline & $\operatorname{HBsAg}(-), n$ & 2535 & 1285 & 3820 & \\
\hline & $(\%, 95 \% \mathrm{Cl})$ & $(98.4,97.9-98.9)$ & $(99.2,98.7-99.7)$ & $(98.7,98.3-99.1)$ & \\
\hline
\end{tabular}


Table 3 Booster response rate and change of overall anti-HBs seropositive rate before and after one booster dose

\begin{tabular}{|c|c|c|c|c|c|c|c|}
\hline \multirow{2}{*}{$\begin{array}{c}\text { Year } \\
\text { [birth cohort] }\end{array}$} & \multicolumn{2}{|c|}{ Seroprevalence, n (\%) } & \multicolumn{2}{|c|}{ Subjects, n (\%) } & \multirow{2}{*}{$\begin{array}{l}\text { Post-booster } \\
\text { Anti-HBs (+) }\end{array}$} & \multicolumn{2}{|c|}{ Anti-HBs titer (median) } \\
\hline & Anti-HBs (+) & Anti-HBs (-) & Booster rate & Response rate & & Before & After \\
\hline \multirow[b]{2}{*}{2007 [1992] } & \multirow[b]{2}{*}{$90(53.6)$} & \multirow[b]{2}{*}{$78(46.4)$} & \multirow{2}{*}{$66(84.6)$} & \multirow{2}{*}{$55(83.3)$} & \multirow{2}{*}{$86.3 \%$} & $2.1 \pm 2.3$ & $399.4 \pm 394.0$ \\
\hline & & & & & & $(\mathrm{P} 50=1.4)$ & $(P 50=288.5)$ \\
\hline \multirow{2}{*}{2008 [1993] } & \multirow{2}{*}{$67(39.4)$} & \multirow{2}{*}{$103(60.6)$} & \multirow{2}{*}{$90(87.4)$} & \multirow{2}{*}{$75(83.3)$} & \multirow{2}{*}{$83.5 \%$} & $1.7 \pm 2.1$ & $480.7 \pm 423.4$ \\
\hline & & & & & & $(P 50=0.7)$ & $(\mathrm{P} 50=365.5)$ \\
\hline \multirow{2}{*}{2009 [1994] } & \multirow{2}{*}{$85(49.4)$} & \multirow{2}{*}{$87(50.6)$} & \multirow{2}{*}{$76(87.4)$} & \multirow{2}{*}{$77(100.0)$} & \multirow{2}{*}{$93.6 \%$} & $1.5 \pm 2.2$ & $678.4 \pm 385.5$ \\
\hline & & & & & & $(\mathrm{P} 50=0.3)$ & $(\mathrm{P} 50=975.0)$ \\
\hline \multirow{2}{*}{2010 [1995] } & \multirow{2}{*}{$61(35.1)$} & \multirow{2}{*}{$113(64.9)$} & \multirow{2}{*}{$96(85.0)$} & \multirow{2}{*}{$85(87.6)$} & \multirow{2}{*}{$83.4 \%$} & $2.7 \pm 2.0$ & $540.2 \pm 421.2$ \\
\hline & & & & & & $(\mathrm{P} 50=2.3)$ & $(\mathrm{P} 50=556.5)$ \\
\hline \multirow{2}{*}{2011 [1996] } & \multirow{2}{*}{$40(23.7)$} & \multirow{2}{*}{$129(76.3)$} & \multirow{2}{*}{$112(86.8)$} & \multirow{2}{*}{$111(99.1)$} & \multirow{2}{*}{$89.3 \%$} & $1.2 \pm 2.0$ & $589.3 \pm 377.2$ \\
\hline & & & & & & $(\mathrm{P} 50=0.2)$ & $(\mathrm{P} 50=555.4)$ \\
\hline \multirow{2}{*}{2012 [1997] } & \multirow{2}{*}{$54(26.9)$} & $147(73.1)$ & $130(884)$ & $126(969)$ & $895 \%$ & $2.6 \pm 2.8$ & $579.2 \pm 387.7$ \\
\hline & & & & (1) & & $(P 50=1.7)$ & $(\mathrm{P} 50=573.7)$ \\
\hline Total & 397 (37.7) & 657 (62.3) & $570(86.8)$ & $529(92.5)$ & $877 \%$ & $2.0 \pm 2.3$ & $551.5 \pm 403.6$ \\
\hline & & & & & & $(\mathrm{P} 50=1.1)$ & $(\mathrm{P} 50=545.5)$ \\
\hline
\end{tabular}

university, grouped as "the same school". The other $1256(97.1 \%)$ subjects of the 18-year age group within the same birth cohort were labeled as "the others", meaning they graduated from another senior high school with unclear booster status. Figure 3 shows that the anti-HBs seropositivity rate of these 38 subjects in "the same school" group at 15 years of age was $31.6 \%(12 / 38)$, while $68.4 \%$ $(26 / 38)$ of them demonstrated double seronegativity of anti-HBs/HBsAg at this age. Among 26 double seronegative subjects, $96.2 \%(25 / 26)$ received one booster dose of $\mathrm{HBV}$ vaccine at age 15 years. The response rate at 6 weeks after the HBV booster among these 25 recipients was $96.0 \%(24 / 25)$. The overall anti-HBs seropositivity rate for all "the same school" subjects at 6 weeks and 3 years after one booster dose was $94.8 \%$ and $76.3 \%$, respectively. Among the 24 recipients showing anti-HBs seroconversion at 6 weeks after booster, seven subjects (29.2\%) had lost their anti-HBs seropositivity again within 3 years. The lone double seronegative subject in "the same school" group who did not receive a booster $(1 / 26)$ retained her double seronegative status for the duration of the 3-year follow-up interval. For those 12 subjects with anti-HBs seropositive

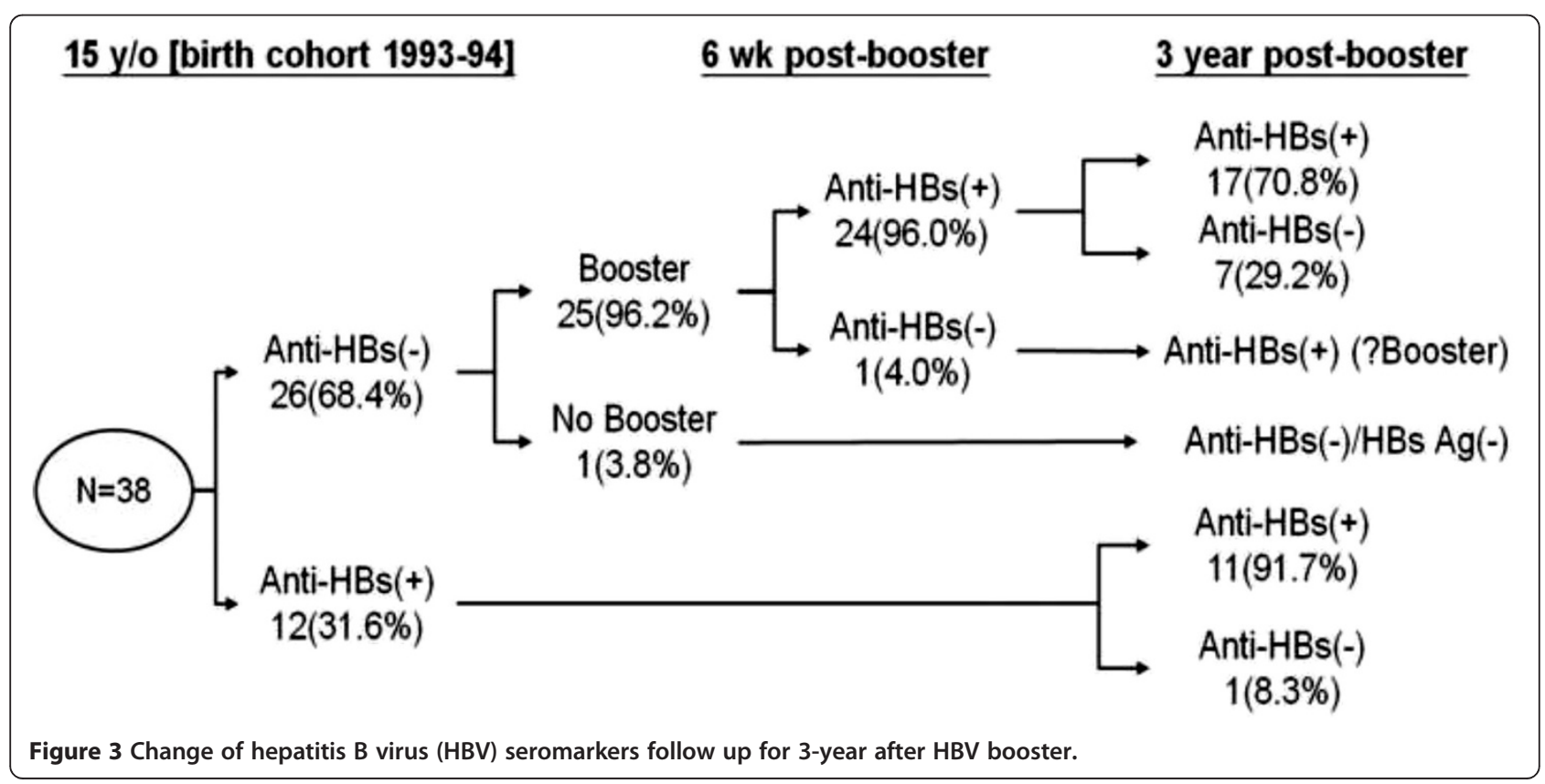


status at 15 years of age, $91.7 \%$ (11/12) of them still retained anti-HBs seropositivity after 3 years.

We also compared the 38 subjects of "the same school" group with 1256 subjects of "the others" group from the birth cohort 1993-1994 in the 18-year age group to determine if higher rates of anti-HBs seropositivity did indeed provide better protection against HBV infection. Table 4 shows that the seropositivity rates of anti-HBs among "the same school" group versus "the others" group were 76.3\% and $39.7 \%$, respectively $(\mathrm{p}<0.001)$. Median titers of antiHBs were also shown to be significantly different between these two groups (i.e., 24.7 vs $4.2 \mathrm{mIU} / \mathrm{mL}$, in "the same school" and "the others", respectively, $\mathrm{p}<0.001)$. There were no new cases of HBsAg seropositivity in "the same school" group, and the seroprevalence rate of $\mathrm{HBsAg}$ was $0.8 \%$ in "the others" group.

\section{Discussion}

In previous studies conducted in Taiwan, the seropositivity rate of anti-HBs declined from $99 \%$ at 1 year of age to $83 \%$ at 5 years of age [15] and further dropped to $71.1 \%$ at 7 years of age, $37.4 \%$ at 12 years of age and $37 \%$ at $15-17$ years of age [16]. The HBsAg seropositivity rate in children less than 12 years of age decreased from $9.8 \%$ in 1984 to $1.3 \%$ in 1994 . The seropositivity rate of anti-HBc also decreased from $26 \%$ in 1984 and $15 \%$ in 1989 to $4 \%$ in 1994 [1]. In a 2004 survey, $\mathrm{Ni}$ et al. showed that the anti-HBc seropositivity rate was low (1\%) in children less than 15 years of age [7]. In 2004, the seropositivity rates for HBsAg, anti-HBs, and anti-HBc were $1.2 \%, 50.5 \%$, and $3.7 \%$, respectively, in those born after implementation of the vaccination program (age $<20$ years) [7].

In our study, the overall seropositivity rates of HBsAg and anti-HBs were $1.2 \%$ and $43.6 \%$, respectively, in those individuals less than 18 years of age. In Table 1, we noted a phenomenon in which the seropositivity rate of antiHBs decayed from $63.4 \%$ (age 6 years) to 31.6\% (age 12 years) and then increased from 12 to 18 years of age. To the best of our knowledge, there is no research reporting a phenomenon similar to the data presented in Figure 2. Reactivation effects (HBV vaccine booster), natural boosters (boosting of the anti-HBs titer after a natural exposure), and breakthrough infections (having HBV infection despite receiving three or more doses of HBV vaccine) could all contribute to the pattern of increase in anti-HBs from 12 to 18 years of age as observed in this study. Tracing our subjects by their birth cohort (1992-1994) in the 12-year age group, we found 110 subjects $(110 / 510,21.6 \%$ of total enrolled students of senior high school in the corresponding period) had also enrolled in our senior high school at 15 years of age. Of subjects with seronegative anti-HBs titers at 12 years of age, 78.8\% $(63 / 80)$ were found to be seropositive at age 15 years (data not shown). We were unable to trace their booster records individually, although such seroconversion was highly indicative of booster effects, which may be a factor that interfered with the anti-HBs seropositivity rate in this birth cohort in the 15-year age group. Similarly, booster effects might also contribute to the elevated seropositivity rate and median titers of anti-HBs in the 18-year age group with less amplitude of influence if compared with those values in the 15-year age group.

In previous studies, the anti-HBc positivity rate of elementary school first graders within the 3-year follow-up period (2005-2007, birth cohort 1999) was between 1 and $2 \%$ in the North, Central, and South of Taiwan but was higher (3.5-4.8\%) in Eastern Taiwan [17]. Another survey conducted in 2004 among children younger than 18 years of age in Taiwan showed breakthrough HBV infection was about $1 \%$ in children sampled (136/13765) [7]. Therefore, the unique phenomenon of increasing anti-HBs seropositivity rate after 12 years of age was likely because of booster reactivation effects. Poovorawan et al. reported that breakthrough infections during the first decade after primary vaccination in infancy were only observed in children born to high-risk families (maternal seropositive for both HBsAg and HBeAg). In the second decade after primary vaccination, breakthrough infections were detected in $12.9 \%$ of individuals, possibly reflecting increased exposure outside the home, linked to high-risk adolescent behaviors [18].

The highest rate of HBsAg seropositivity was $1.6 \%$ in the 12-year age group of this study, but no statistical significance $(\mathrm{p}=0.154)$ was observed in the pattern of

Table 4 Comparison of seroprevalence of HBsAg and anti-HBs in "the same school" and "the others" groups

\begin{tabular}{cccc}
\hline \multirow{2}{*}{ Item } & \multicolumn{1}{c}{ 18-year age group (birth cohort 1993-1994) } \\
\cline { 2 - 4 } & The same school $(\mathbf{n}=\mathbf{3 8})$ & The others $(\mathbf{n}=\mathbf{1 2 5 6 )}$ & P-value \\
\hline Anti-HBs $(+), \mathrm{n}(\%, 95 \% \mathrm{Cl})$ & $29(76.3,62.8-89.8)$ & $499(39.7,37.0-42.4)$ & $757(60.3,57.6-63.0)$ \\
Anti-HBs $(-), \mathrm{n}(\%, 95 \% \mathrm{Cl})$ & $9(23.7,10.2-37.2)$ & $58.8 \pm 145.9(\mathrm{P} 50=4.2)$ & $10(0.8 \%)$ \\
Anti-HBs titer, mean \pm SD (median) & $157.6 \pm 281.3(\mathrm{P} 50=24.7)$ & $1246(99.2 \%)$ & $<0.001^{*}$ \\
HBsAg (+), $\mathrm{n}(\%)$ & $0(0.0)$ & $38(100.0)$ & $0.001^{*}$ \\
HBsAg $(-), \mathrm{n}(\%)$ & &
\end{tabular}

Data are presented as $\mathrm{n}$ and percentage or mean \pm standard deviation (median).

*p-value $<0.05$ was considered statistically significant after test. 
anti-HBs seroprevalence by age. All students in the 12year age group were born between 1992 and 1994 and their HBsAg seropositivity rate was consistent with other studies within the same time frame [17]. Natural HBV infections (positive for anti-HBc) and $\mathrm{HBV}$ carriers (seropositive HBsAg) were found in $4.1 \%$ and $1.6 \%$ of individuals, respectively, at 15-17 years of age in a post1986 cohort in Taiwan [10].

Based on data from previous studies, different types of $\mathrm{HB}$ vaccines, doses, and brands as well as the timing of primary vaccination can all influence the persistence of anti-HBs titers [19]. In Taiwan, neonates born before July 1992 received four doses of plasma-derived vaccine (Hevac B; Pasteur-Merieux, or its equivalent derivative); neonates born after July 1992 received recombinant HBV vaccines $(5 \mu \mathrm{g} /$ dose of Recombivax [Merck] or $20 \mu \mathrm{g} /$ dose of Engerix [GSK]) instead. In Table 2, we demonstrate that individuals in the plasma-derived subgroup had a higher proportion of positivity for anti-HBs than did those of the recombinant subgroup in both the 15 - and 18-year age groups (43.2\% vs $34.7 \%$ at 15 years, $\mathrm{p}<0.004 ; 46.0 \%$ vs $40.8 \%$ at 18 years, $\mathrm{p}<0.003)$. Our finding is consistent with several other studies [20,21]. The seropositivity rate of $\mathrm{HBsAg}$ in the plasma-derived subgroup is significantly higher than that of the recombinant subgroup in the 18-year age group $(\mathrm{p}=0.049)$ alone, but not in the 15 -year age group $(p=0.131)$. In our subsequent study, only the subjects who received recombinant $\mathrm{HBV}$ vaccine were pooled together for a longitudinal study to avoid the interference of different vaccine type and dosage given in infancy.

According to the birth cohort (1987-2003) analysis for long-term immunity following infant $\mathrm{HBV}$ vaccination, no increase of the seropositivity rates of HBsAg and anti-HBc was noted after 17 years of age [7]. In contrast, $15-50 \%$ of the children who initially responded to the three-dose series of HBV vaccination had low or undetectable antiHBs levels 5-15 years after primary vaccination [8]. Wu et al. reported that the overall anti-HBs seropositivity rate after a booster dose of $\mathrm{HB}$ vaccine was estimated to be 84.3\% among 1974 students from senior high schools with negative titers of both anti-HBs and $\mathrm{HBsAg}$ before the $\mathrm{HB}$ vaccine booster [13]. Long-term protection studies indicated that immunological memory usually persisted even if anti-HBs levels fell below the protective threshold $(10 \mathrm{mIU} / \mathrm{mL})$ [20,21]. However, without protective levels of anti-HBs, memory cells alone are probably unable to protect against acute infection [22]. In our subjects within the 15-year age group from the birth cohort 1992-1997 presented in Table 3, booster response rate was $92.5 \%$ (529/570) after one booster dose of recombinant HBV vaccine $(20 \mu \mathrm{g} /$ dose $)$. Anti-HBs seropositivity rate before one booster dose was 37.7\% (397/1054) and became $87.7 \%$ afterwards. The median titers of anti-HBs before and after booster dose were $1.1 \mathrm{mIU} / \mathrm{mL}$ and $545.5 \mathrm{mIU} / \mathrm{mL}$, respectively. All the subjects received recombinant HBV vaccine in their neonatal period, except the individuals from the 1992 birth cohort, who received plasma-derived HBV vaccine if they were born before July 1992. Lu et al. showed the booster response rate to one dose of recombinant $\mathrm{HBV}$ vaccine was $71 \%$ in 15 - to 17 -year-old subjects who received plasma-derived $\mathrm{HBV}$ vaccine during neonatal immunization [10]. All the subjects from the $\mathrm{Wu}$ et al. [13] study were born between July 1987 and July 1991 and thus belonged to the plasma-derived HBV vaccine era in Taiwan. Therefore, the booster response pointed to the recipients who received the plasma-derived vaccine in infancy.

In Figure 3, we selected and followed 38 subjects who had graduated from our senior high school and consequently studied in our university. We monitored the changes in the seropositivity rates of anti-HBs and HBsAg within a time frame of 3 years. We found that the initial booster response rate at 6 weeks after one booster dose of $\mathrm{HBV}$ vaccine was as high as $96 \%(24 / 25)$ in our 15 -yearold subjects. Surprisingly, seven subjects (29.2\%, 7/24 booster responders) who became seropositive after their booster dose lost their anti-HBs seropositivity again within 3 years after booster. The overall anti-HBs seropositivity rate for all "the same school" subjects at 6 weeks and 3 years after one booster dose was $94.8 \%$ and $76.3 \%$, respectively. For the comparison group labeled as "the others", subjects from the same birth cohort in the 18-year age group, the seropositivity rates of anti-HBs and HBsAg were $39.7 \%(499 / 1256)$ and $0.8 \%$ (10/1256), respectively. The median titer of subjects in "the others" group was $4.2 \mathrm{mIU} / \mathrm{mL}(24.7 \mathrm{mIU} / \mathrm{mL}$ in "the same school"). During the 3-year follow-up period, no new HBV carriers were detected in "the same school" group, which included students given a booster dose if their antiHBs titers were seronegative. However, there was no statistical difference $(\mathrm{p}=0.581)$ in HBV carrier rate between "the same school" subgroup and "the others" subgroup ( $0 \%$ vs $0.8 \%$, respectively). An HBsAg seropositivity rate of $0.8 \%$ at 18 years of age in our study group was comparable to the seroprevalence of the same age group in other studies [7]. In our study, booster vaccination did not confer additional protection against HBsAg carriage. High booster response rate (96\%) at 6 weeks after one booster dose at 15 years of age also indicated intact long-term protection of $\mathrm{HBV}$ infection by immunological memory, as a result of anamnestic response after primary infant $\mathrm{HBV}$ vaccination. Immunity against $\mathrm{HBV}$ provided protection against infection as well as against disease. Protection against infection is associated with antibody persistence, which is directly related to the peak production of antiHBs after primary vaccination. Protection against disease (i.e., acute hepatitis, prolonged viraemia, carriership, and 
chronic infection) is associated with immune memory that persists beyond the time at which anti-HBs disappears [9]. Currently, Middleman et al. show several variables independently associated with higher geometric mean titer response to a challenge dose of vaccine included a higher baseline anti-HBs titer, older age at first dose of primary series ( $\geq 4$ weeks after birth), higher test dosage, and nonwhite race [23]. In contrast to the high endemicity of HBV in Taiwan, Middleman et al. collected these new data regarding duration of protection in the setting of low hepatitis B endemicity in the United States, with a likely absence of natural boosting. The response to the challenge dose of $\mathrm{HB}$ vaccine was remarkably good, as high as $92 \%$ [23], which is similar to our booster response rate after one booster dose of $\mathrm{HBV}$ vaccine at 15 years of age.

Taking Taiwan as an example of an HBV endemic area, the seropositivity rates of $\mathrm{HBsAg}$ and $\mathrm{HBeAg}$ for pregnant women were still $10.3 \%$ and $2.3 \%$ in 2009 (data from Taiwan Centre for Disease Control, Department of Health). Additionally, the mean age of mothers giving birth in 2007-2009 was 29.45 years of age, which meant the majority were born after the introduction of infant HBV vaccination. Horizontal and breakthrough infection could also occur after waning or eventual loss of the vaccine protectiveness in older children, especially with changes in lifestyle and sexual activity [9]. A concern exists about sexual exposure to HBsAg carriers in hyperendemia areas such as Taiwan when vaccinated children become adolescents and young adults. Ni et al. showed no increase in seropositivity rates of either HBsAg or anti-HBc when vaccinated individuals progressed to 17 years of age [7] and the rate of chronicity declined as the age of infection increased: $25 \%$ in infected preschool children and $3-10 \%$ in adolescents and young adults [24]. Although universal booster dose may not be necessary up to 20 years after the primary vaccination because HBsAg and anti-HBc seropositivity did not increase [7], the methods for the prevention of horizontal transmission, such as avoidance of skin tattooing, use of disposable needles, and condom use in sexual contact still need continuous implementation in the adolescent group [25]. Based on our data, the current guidelines from Taiwan Advisory Committee on Immunization Practice (ACIP) appear to be adequate, and states that individuals may receive a booster dose if they have negative anti-HBs antibodies and are in high-risk groups (i.e., hemodialytic, organ transplant, and immunocompromised patients; intravenous drug users; participants in high-risk sexual activity; or health care workers).

The major limitations of our study were as follows. First, the retrospective cross-sectional study design and records analysis was conducted without reviewing any vaccination records for all subjects. Second, quantitative data of antiHBs titers were available for all four age groups in the period of 2007-2012 but anti-HBc was not routinely screened for during entrant health-screening examination owing to a decreased seropositivity rate of this marker $[26,27]$ and a high proportion of HBV-DNA negativity in anti-HBc positive subjects [28]. As a result, the seropositivity rates of natural infection could not be estimated in our study. Third, despite achievement of high HBV vaccine coverage rates from $88.8 \%$ to $96.9 \%$ (from 1984 to 2010) according to data from the Centre for Disease Control in Taiwan [11,12], there was a possible bias in primary versus booster doses in our study subjects. The proportion of booster rate in "the others" subgroup analysis could not be identified and therefore we could only state possible booster dose effects according to the level of anti-HBs titers when comparing these data to the value of anti-HBs in subjects from the same birth cohort.

\section{Conclusions}

HBV booster strategy at 15 years of age was the main contributor to the unique age-related phenomenon of anti-HBs seropositive rate and titers. No increase in HBsAg seropositivity rates within different age groups $(p=0.154)$ was observed. Female individuals demonstrated significantly higher anti-HBs seropositivity compared with male individuals $(\mathrm{p}<0.015)$. Vaccination with plasma-derived HBV vaccines in infancy provided a higher rate of anti-HBs seropositivity at 15-18 years of age in our study than the recombinant vaccine. The overall response rate at 6 weeks after one HBV booster dose was $92.5 \%$ in subjects demonstrating double seronegativity for anti-HBs and HBsAg, which indicated that intact immunogenicity persisted at least 15 years after primary infant $\mathrm{HBV}$ vaccination. Booster vaccination of HBV did not confer additional protection against HBsAg carriage in our study. Therefore, based on our data, we conclude that the current booster strategy implemented by Taiwan ACIP, which states that individuals may receive a booster dose if they have negative anti-HBs antibodies and are in high-risk groups (i.e., hemodialytic, organ transplant, and immunocompromised patients; intravenous drug users; participants in high-risk sexual activity; or health care workers), remains adequate.

\section{Competing interests}

The authors declare that they have no competing interests.

\section{Authors' contributions}

YCC conceived of the study, and participated in its design, the integrity of the data and the accuracy of the data analysis. JHW participated in the data analysis, statistical analysis and drafted the tables and figures. YSC and JSL carried out the acquisition of raw data. CFC participated in the design of the study. CHC participated in its design, the integrity and interpretation of the data. All authors read and approved the final manuscript.

\section{Acknowledgements}

We thank the Department of Medical Affairs for organization the school children heath examination and all doctors in Department of Pediatrics, Family Medicine, and Dentistry in Hualien Tzu Chi Hospital, Buddhist Tzu Chi 
Medical Foundation for their devotion on time and works. We also thank the Ministry of Education for their assistant.

\section{Author details}

'Department of Pediatrics, Hualien Tzu Chi Hospital, Buddhist Tzu Chi Medical Foundation, Hualien, Taiwan. ${ }^{2}$ Department of Medical Research, Hualien Tzu Chi Hospital, Buddhist Tzu Chi Medical Foundation, Hualien, Taiwan. ${ }^{3}$ Institute of Biomedical Sciences, Academia Sinica, Taipei, Taiwan.

Received: 30 March 2014 Accepted: 18 September 2014

Published: 23 September 2014

\section{References}

1. Chen HL, Chang MH, Ni YH, Hsu HY, Lee PI, Lee CY: Seroepidemiology of hepatitis $B$ virus infection in children: ten years of mass vaccination in Taiwan. JAMA 1996, 276:906-908

2. $\mathrm{Ni} \mathrm{YH}$, Chen DS: Hepatitis B vaccination in children: the Taiwan experience. Pathol Biol 2010, 58:296-300.

3. Jack AD, Hall AJ, Maine N, Mendy M, Whittle HC: What level of hepatitis B antibody is protective? I Infect Dis 1999, 179:489-492.

4. Hsu HY, Chena MH, Chen DS, Lee CY, Sung JL: Baseline seroepidemiology of hepatitis B virus infection in children in Taipei: a study just before mass hepatitis B vaccination program in Taiwan. J Med Virol 1986, 18:301-307.

5. Tsen YJ, Chang MH, Hsu HY, Lee CY, Sung JL, Chen DS: Seroepidemiology of hepatitis B virus infection in Taipei, 1989-five years after a mass hepatitis B virus vaccination program. J Med Virol 1991, 34:96-99.

6. $\mathrm{Ni}$ YH, Chang MH, Huang LM, Chen HL, Hsu HY, Chiu TY: Hepatitis B virus infection in children and adolescents in a hyperendemic area: 15 years after universal hepatitis B vaccination. Ann Intern Med 2001, 135:796-800

7. $\mathrm{Ni}$ YH, Huang LM, Chang MH, Yen CJ, Lu CY, You SL: Two decades of universal hepatitis B vaccination in Taiwan: impact and implication for future strategies. Gastroenterology 2007, 132:1287-1293.

8. Plotkin S, Orsenstein W, Offit P: Vaccines. Philadelphia: Elsevier; 2008.

9. Lu CY, Chiang BL, Chi WK: Waning immunity to plasma-derived hepatitis $B$ vaccine and the need for boosters 15 years after neonatal vaccination. Hepatology 2004, 40:1415-1420.

10. Lu CY, Ni YH, Chiang BL: Humoral and cellular immune responses to a hepatitis B vaccine booster 15-18 years after neonatal immunization. $J$ Infect Dis 2008, 197:1419-1426.

11. Chien YC, Jan CF, Kuo HS, Chen CJ: Nationwide hepatitis B vaccination program in Taiwan: effectiveness in the 20 years after it was launched. Epidemiol Rev 2006, 28:126-135.

12. Centers for Disease Control, Department of Health, ROC (Taiwan): CDC: 2012 Annual Report. http://www.cdc.gov.tw/uploads/files/201208/67addf76-04934d7b-aa72-cd51 beff6654.pdf. Accessed February 17, 2013

13. Wu TW, Li HH, Wang LY: Chronic hepatitis B infection in adolescents who received primary infantile vaccination. Hepatology 2013, 57:37-45.

14. European Consensus Group on Hepatitis B Immunity: Are booster immunisations needed for lifelong hepatitis B immunity? Lancet 2000, 355:561-565.

15. Lee PI, Lee $C Y$, Huang LM, Chang MH: Long-term efficacy of recombinant hepatitis $B$ vaccine and risk of natural infection in infants born to mothers with hepatitis B e antigen. J Pediatr 1995, 126:716-721.

16. Lin YC, Chang MH, Ni YH, Hsu HY, Chen DS: Long-term immunogenicity and efficacy of universal hepatitis B virus vaccination in Taiwan. J Infect Dis 2003, 187:134-138.

17. Chen CY, Hsu HY, Liu CC, Chang MH, Ni YH: Stable seroepidemiology of hepatitis B after universal immunization in Taiwan: a 3-year study of national surveillance of primary school students. Vaccine 2010, 28:5605-5608.

18. Poovorawan Y, Chongsrisawat C, Theamboonlers A, Leroux-Roels G, Kuriyakose $S$, Leyssen M, Jacquet JM: Evidence of protection against clinical and chronic hepatitis B infection 20 years after infant vaccination in a high endemicity region. J Viral Hepat 2011, 18(5):369-375.

19. Koff RS: Immunogenicity of hepatitis B vaccines: implications of immune memory. Vaccine 2002, 20:3695-3701.

20. Poovorawan $Y$, Chongsrisawat V, Theamboonlers A, Bock HL, Leyssen M, Jacquet JM: Persistence of antibodies and immune memory to hepatitis B vaccine 20 years after infant vaccination in Thailand. Vaccine 2010, 28:730-736.
21. Wu Q, Zhuang GH, Wang XL, Wang LR, Li N, Zhang M: Antibody levels and immune memory 23 years after primary plasma-derived hepatitis $B$ vaccination: results of a randomized placebo-controlled trial cohort from China where endemicity is high. Vaccine 2011, 29:2302-2307.

22. Amanna IJ, Slifka MK: Contributions of humoral and cellular immunity to vaccine-induced protection in humans. Virology 2011, 411:206-215.

23. Middleman AB, Baker CJ, Kozinetz CA, Kamili S, Nguyen C, Hu DJ, Spradling PR: Duration of protection after infant hepatitis $B$ vaccination series. Pediatrics 2014, 133(6):e1500-e1507.

24. Chang MH: Hepatitis B virus infection. Semin Fetal Neonatal Med 2007, 12:160-167.

25. Hsu SC, Chang MH, Ni YH, Hsu HY, Lee CY: Horizontal transmission if hepatitis B virus in children. J Pediatr Gastroenterol Nutr 1993, 16:66-69.

26. Sua FH, Cheng SH, Li CY, Chen JD, Hsiao CY, Chien CC: Hepatitis B seroprevalence and anamnestic response amongst Taiwanese young adults with full vaccination in infancy, 20 years subsequent to national hepatitis B vaccination. Vaccine 2007, 25:8085-8090.

27. Lin DB, Wang HM, Lee YL, Ling UP, Changlais SP, Chen CJ: Immune status in preschool children born after mass hepatitis $B$ vaccination program in Taiwan. Vaccine 1998, 16:1683-1687.

28. Altunay H, Kosan E, Birinci I, Aksoy A, Kirali K, Saribas S: Are isolated anti-HBC blood donors in high risk group? The detection of HBV DNA in isolated anti-HBC cases with nucleic acid amplification test (NAT) based on transcription mediated amplification (TMA) and HBV discrimination. Transfus Apheresis Sci 2010, 43:265-268.

doi:10.1186/1471-2458-14-991

Cite this article as: Chang et al:: Hepatitis B virus vaccination booster does not provide additional protection in adolescents: a cross-sectional school-based study. BMC Public Health 2014 14:991.

\section{Submit your next manuscript to BioMed Central and take full advantage of:}

- Convenient online submission

- Thorough peer review

- No space constraints or color figure charges

- Immediate publication on acceptance

- Inclusion in PubMed, CAS, Scopus and Google Scholar

- Research which is freely available for redistribution 